\title{
Tevatron Performance Goals for the Coming Decade
}

\author{
Stephen D. Holmes \\ Fermi National Accelerator Laboratory \\ P.O. Box 500, Batavia, Illinois 60510
}

April 1999

Presented at PAC99, Particle Accelerator Conference, New York, March 29-April 2, 1999 


\section{Disclaimer}

This report was prepared as an account of work sponsored by an agency of the United States Government. Neither the United States Government nor any agency thereof, nor any of their employees, makes any warranty, express or implied, or assumes any legal liability or responsibility for the accuracy, completeness, or usefulness of any information, apparatus, product, or process disclosed, or represents that its use would not infringe privately owned rights. Reference herein to any specific commercial product, process, or service by trade name, trademark, manufacturer, or otherwise, does not necessarily constitute or imply its endorsement, recommendation, or favoring by the United States Government or any agency thereof. The views and opinions of authors expressed herein do not necessarily state or reflect those of the United States Government or any agency thereof.

\section{Distribution}

Approved for public release; further dissemination unlimited.

\section{Copyright Notification}

This manuscript has been authored by Universities Research Association, Inc. under contract No. DE-AC02-76CH0300O with the U.S. Department of Energy. The United States Government retains and the publisher, by accepting the article for publication, acknowledges that the United States Government retains a nonexclusive, paid-up, irrevocable, worldwide license to publish or reproduce the published form of this manuscript, or allow others to do so, for United States Government Purposes. 


\title{
TEVATRON PERFORMANCE GOALS FOR THE COMING DECADE
}

\author{
Stephen D. Holmes, Fermi National Accelerator Laboratory*, P.O. Box 500, Batavia, IL 60510
}

\section{Abstract}

Fermilab is in the midst of a program to raise the luminosity in the Tevatron proton-antiproton collider by at least a factor of five above the currently achieved level of $1.6 \times 10^{31} \mathrm{~cm}^{-2} \mathrm{sec}^{-1}$. Components of this program include the construction of a new synchrotron, the Main Injector, a new antiproton storage ring, the Recycler, and a variety of improvements to the existing Antiproton Source and Tevatron. Commissioning of these components will be underway in early 1999 with the next Tevatron collider run scheduled to start in April 2000. Initial commissioning experience with these systems will be described, along with developments underway to support further Tevatron luminosity enhancements over the coming decade.

\section{PROGRAM GOALS}

The Fermilab Tevatron is the highest energy collider operating in the world today and will remain so until the initiation of LHC operations. The goal of the Tevatron collider program over the upcoming decade is to exploit the capabilities of this facility to the fullest extent possible while it retains this unique position. In support of this goal a new proton accelerator, the Fermilab Main Injector, and a new antiproton storage ring, the Recycler Ring, have recently been constructed. These facilities are now nearing completion and will support reinitiation of Tevatron collider operations in early 2000.

Table 1 lists operational parameters achieved in the most recent collider run, "Run IB", and those expected in the upcoming run, "Run II". Two sets of Run II parameters are presented: one based on operations with 36 proton and 36 antiproton bunches and a second based on 140 proton and 121 antiproton bunches. Bunch configurations during Run II will be chosen to maintain fewer than three interactions per crossing as seen by the two experimental detectors. Initial operations during Run II will be with the $36 \times 36$ bunch configuration, rising to $140 \times 121$ as the luminosity surpasses $1 \times 10^{32} \mathrm{~cm}^{-2} \mathrm{sec}^{-1}$. The primary source of improved performance relative to Run IB is an increase in the number of antiprotons in the collider, directly attributable to the Main Injector. Achievement of luminosities beyond $1 \times 10^{32} \mathrm{~cm}^{-2} \mathrm{sec}^{-1}$ are expected once the recovery of antiprotons at the ends of stores is implemented. The initial programmatic goal for Run II is to deliver $2 \mathrm{fb}^{-1}$ of integrated luminosity by the end of 2002 .

\footnotetext{
"Operated by University Research Association under contract to the U.S. Department of Energy
}

Table 1: Tevatron Collider operational parameters achieved in the most recent collider run (Run IB) and expected in the upcoming run (Run II).

\begin{tabular}{|l|r|r|r|l|}
\hline RUN & IB & II $(36 \times 36)$ & II $(140 \times 121)$ & \\
\hline \hline Protons/bunch & $2.3 \times 10^{11}$ & $2.7 \times 10^{11}$ & $2.7 \times 10^{11}$ & \\
\hline Pbars/bunch & $5.5 \times 10^{10}$ & $3.0 \times 10^{10}$ & $3.0 \times 10^{10}$ & \\
\hline Total Pbars & $3.3 \times 10^{11}$ & $1.1 \times 10^{12}$ & $3.6 \times 10^{12}$ & \\
\hline Pbar Production Rate & $6.0 \times 10^{10}$ & $2.0 \times 10^{11}$ & $2.0 \times 10^{11}$ & \\
\hline Proton Emittance & $23 \pi$ & $20 \pi$ & $20 \pi$ & $\mathrm{mm}-\mathrm{mr}$ \\
\hline Pbar Emittance & $13 \pi$ & $15 \pi$ & $15 \pi$ & $\mathrm{mm}-\mathrm{mr}$ \\
\hline$\beta^{*}$ & 0.35 & 0.35 & 0.35 & $\mathrm{~m}$ \\
\hline Energy & 900 & 1000 & 1000 & $\mathrm{GeV}$ \\
\hline Bunches & 6 & 36 & 121 & \\
\hline Bunch Length & 0.60 & 0.37 & 0.37 & $\mathrm{~m}$ \\
\hline Crossing Angle & 0 & 0 & 136 & $\mu \mathrm{rad}$ \\
\hline & & & & \\
\hline Luminosity & $1.6 \times 10^{31}$ & $3.6 \times 10^{31}$ & $1.6 \times 10^{32}$ & $\mathrm{~cm}^{-2} \mathrm{~s}^{-1}$ \\
\hline Integrated Luminosity & 3.2 & 17.3 & 32.5 & $\mathrm{pb}^{-1} / \mathrm{week}$ \\
\hline Bunch Spacing & 3500 & 396 & 132 & $\mathrm{nscc}$ \\
\hline Interactions/crossing & 2.5 & 2.3 & 1.3 & \\
\hline
\end{tabular}




\section{ELEMENTS OF RUN II}

The luminosity in the Tevatron is given by the expression,

$$
L=\frac{3_{p f N}\left(B N_{\bar{p}}\right)}{\beta *\left(\varepsilon_{p}+\varepsilon_{\bar{p}}\right)} F\left(\sigma_{z} / \beta^{*}, \phi\right)
$$

where $\gamma$ is the relativistic factor, $f$ is the revolution frequency, $B$ is the number of bunches in each beam, $N_{p}$ $\left(\mathrm{N}_{\mathrm{p}}\right)$ is the number of protons (antiprotons) in a bunch, $\varepsilon_{p}\left(\varepsilon_{p}^{-}\right)$is the $95 \%$ normalized transverse beam emittance, $\sigma_{Z}$ is the rms bunch length, $\beta^{*}$ is the beta function at the interaction point, and $\mathrm{F}$ is a form factor dependent on the ratio of the bunch length to $\beta^{*}$ and to the crossing angle, $\phi$. Potential performance limitations are related to the number of protons per bunch, $\mathrm{Np}$. and the total number of antiprotons in the collider, $\mathrm{BN}_{\overline{\mathrm{p}}}$. While the number of protons per bunch is limited due to beam-beam effects, the primary performance limitation in the Tevatron has been, and will remain, the availability of antiprotons. The current round of modifications to the Tevatron complex are aimed at providing more antiprotons to the collider. The scope of changes to the complex includes construction of the Main Injector and Recycler, and modifications to the Antiproton Source and Tevatron.

\subsection{Main Injector and Recycler}

The Main Injector is a 3319 meter circumference, 150 $\mathrm{GeV}$, conventional-magnet-based accelerator [1]. The Main Injector replaces the recently decommissioned Main Ring accelerator. The Main Injector will fill all duties previously assigned to the Main Ring with significantly improved performance. In particular the Main Injector has been constructed to provide an improved (by at least a factor of three) antiproton production targeting rate, a modest increase in the proton bunch intensity, and a new capability for delivery of high intensity $\left(>3 \times 10^{13}\right.$ protons every $1.9 \mathrm{sec}$.) $120 \mathrm{GeV}$ protons to support fixed target experiments.

The Reycler is a 3319 meter, $8.9 \mathrm{GeV}$, permanentmagnet-based antiproton storage ring [2]. Construction of the Recycler was formally incorporated into the Main Injector project in April 1997. The Recycler will become the third antiproton storage ring supporting the Tevatron complex. The Recycler has been constructed to relieve the existing Antiproton Accumulator of responsibility for maintaining high stacking rates at high stacks, to double the cffective antiproton stacking rate through the recovery of unspent antiprotons from the collider, and to provide a platform for further improvements to the complex. As constructed the Recycler Ring will utilize stochastic cooling and should be capable of supporting stacks containing $3 \times 10^{12}$ antiprotons and a stacking rate of $2 \times 10^{11}$ antiprotons/hour. An electron cooling research and development program is currently underway to support future performance enhancements.

\subsection{Antiproton Source}

Significant modifications have been undertaken to the Antiproton Source to capitalize on the increased intensity delivered from the Main Injector for antiproton production. The goal is to achieve a stacking rate of $2 \times 10^{11}$ protons per hour for stacks containing up to approximately $4 \times 10^{11}$ antiprotons. Modifications to achieve this performance include: 1)doubling the bandwidth of the Accumulator stack-tail stochastic cooling system to $2-4 \mathrm{GHz}$; 2)increasing the transition gamma of the Accumulator by one unit (to 6.4), to accommodate this increase in the cooling bandwidth [3]; and 3)doubling the bandwidth of the Debuncher stochastic cooling system to $4-8 \mathrm{GHz}$, accompanied by the introduction of cryogenically cooled amplifiers [4].

\subsection{Tevatron}

The Tevatron has been reconfigured to support the experimental program enabled hy the new Main Injector and Recycler rings. Primary modifications include: 1)a modest number of magnet replacements and relocations aimed at supporting $1 \mathrm{TeV}$ beam operations; 2)introduction of new injection equipment to accommodate relocation of Tevatron injection from E-0 to F-0 and 36 bunch operations; and 3)construction of a new experimental hall at $\mathrm{C}-0$ for possible future experiments.

\section{INSTALLATION AND COMMISSIONING STATUS}

Figure 1 shows the Main Injector enclosure with the Main Injector installed near the floor and the Recycler installed near the ceiling. The Main Injector installation is now complete. Recycler installation is currently in its final phase with completion scheduled for early April. At the time this is written the entire Recycler ring is installed and under vacuum, with work on the abort line and instrumentation in process. Installation of the stochastic cooling systems is scheduled for late in 1999.

The Antiproton Accumulator lattice modification is complete and the increased bandwidth stack-tail cooling system will be installed by early April. Debuncher cooling system fabrication will continue through the summer with installation late in 1999.

The Tevatron has been reconfigured to support the new beam transfers at F-0. Cooldown of the Tevatron will be initiated in March in anticipation of $800 \mathrm{GeV}$ fixed target run startup in mid-April. A changeover from fixed target to collider configuration will be required following completion of the fixed target run later this year. 


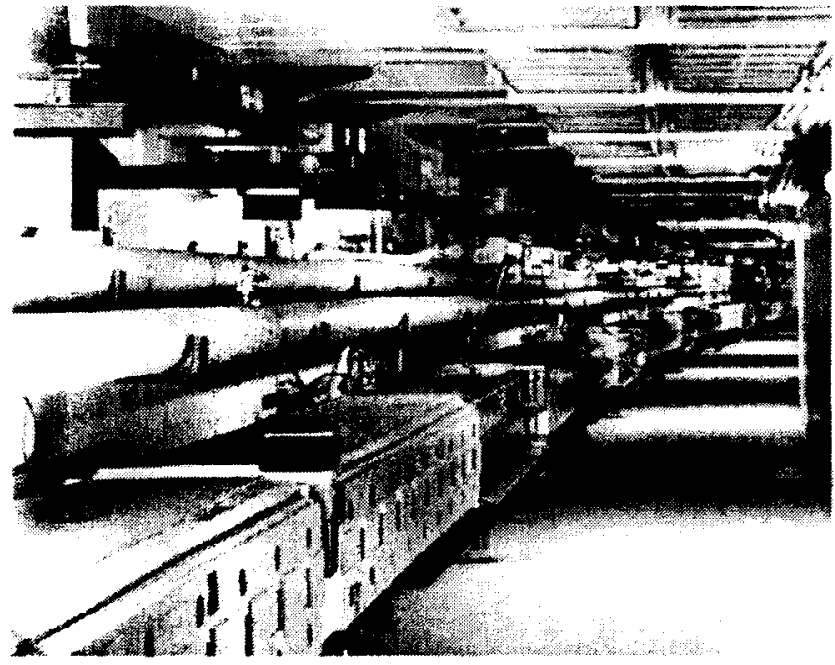

Figure 1: The Main Injector enclosure showing the installed Main Injector (lower) and the permanentmagnet-based Recycler (upper).

\subsection{Main Injector and Recycler Commissioning}

Main Injector commissioning with beam was formally initiated on September 26, 1998 with the first transmission of protons from the Booster through the $8 \mathrm{GeV}$ transfer line and into the Main Injector injection channel. Beam was transmitted through the first two half-cells at which point it was absorbed. The first attempt at circulating beam at the $8 \mathrm{GeV}$ injection energy was made on October 10. Circulating beam was achieved within one hour of the initiation of beam transfers from the Booster. Figure 2 shows the first beam orbit observed in the Main Injector. This achievement was followed by acceleration of beam to the $120 \mathrm{GeV}$ antiproton production energy on November 15 and to the $150 \mathrm{GeV}$ Tevatron injection energy on November 23. Figure 3 shows a typical 150 $\mathrm{GeV}$ acceleration cycle achieved in early January 1999. Six Booster batches are injected and a total intensity slightly in excess of $1 \times 10^{13}$ protons is accelerated to 150 GeV.

In parallel with these efforts beam transfers from the Main Injector to the Antiproton Source and the partially installed Recycler ring were also initiated in January. 8 $\mathrm{GeV}$ protons were transported through approximately onethird the circumference of the Recycler to a temporary heam absorher on January 11, and the first transfer of 8 $\mathrm{GeV}$ beam from the Main Injector to the Antiproton Source was accomplished on January 26.

The Main Injector and Recycler are currently shutdown for completion of the final Recycler installation phase. Current performance may be summarized as follows:

- $1.6 \times 10^{13}$ protons routinely accelerated to $120 \mathrm{GeV}$ with $90 \%$ acceleration efficiency. This is to be compared with the full design goal of $3 \times 10^{13}$.

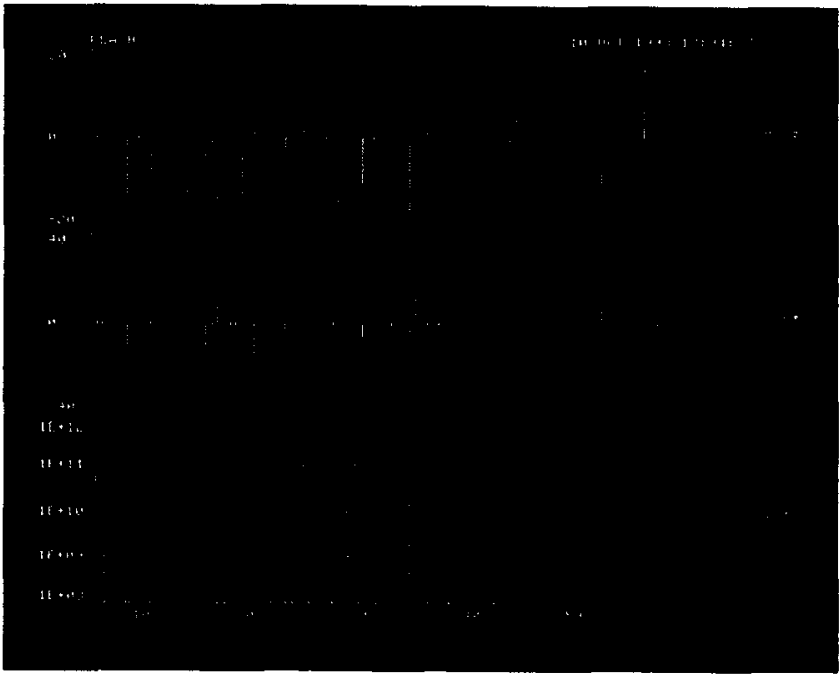

Figure 2: Main Injector beam orbit for the first circulating beam observed at the $8 \mathrm{GeV}$ injection energy. The horizontal orbit position is given on top, vertical position in the middle, and beam intensity on the bottom.

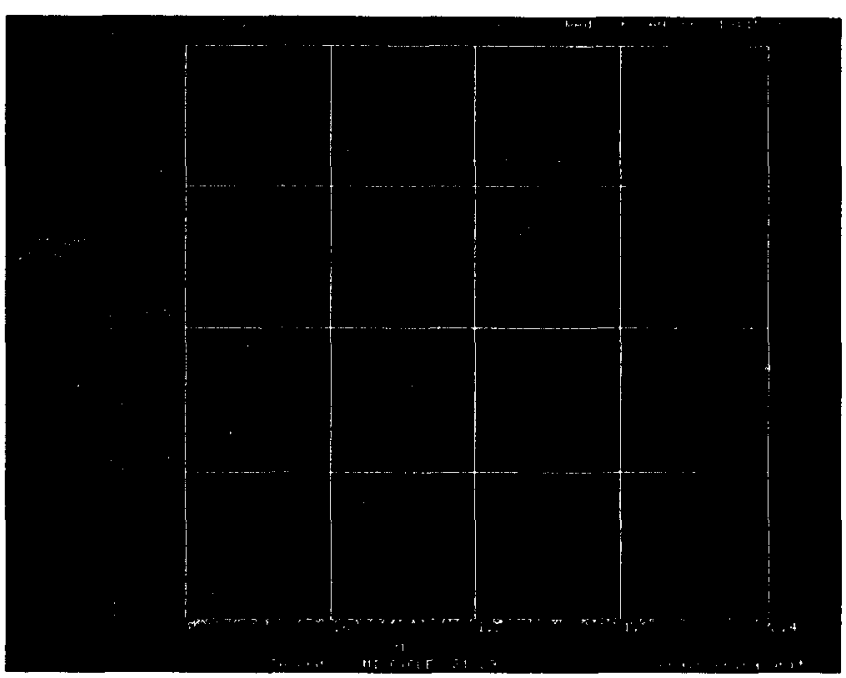

Figure 3: Typical acceleration of protons to $150 \mathrm{GeV}$ during early commissioning of the Main Injector. A total beam intensity of slightly more than $1 \times 10^{13}$ protons is injected over six Booster acceleration cycles and accelerated to the full design energy of $150 \mathrm{GeV}$.

- The full design operating energy of the Main Injector, $150 \mathrm{GeV}$, has been achieved with beam.

- Antiproton targeting cycles with beam have been run with an average repetition rate of 2.5 seconds. The full design goal is 1.5 seconds.

- Low intensity proton beams have been resonantly extracted from the Main Injector.

- The Main Injector optics are well understood with excellent agreement between measured and modeled lattice functions. 
Operations of the Main Injector and first circulating beam commissioning of the Recycler are expected to recommence in early April. At that time we expect to bring the Main Injector up to its full operational performance goals over a period of several months.

\subsection{Antiproton Source Installation and Commissioning}

The reconfigured Antiproton Source is currently being installed. However, commissioning of the new Antiproton Accumulator lattice was initiated in January 1999 without the new stochastic cooling systems completely installed. Proton beams delivered from the Main Injector have been used to recommission the Accumulator as a storage ring with observed beam lifetimes consistent with vacuum lifetime. Currently the Antiproton Source is shutdown with complete installation of the upgraded Accumulator stack-tail cooling systems scheduled for early April, and the upgraded Debuncher cooling system scheduled for fall. Commissioning of the Antiproton Source is expected to recommence in April and continue through 1999.

\subsection{Tevatron Installation and Commissioning}

Tevatron reconfiguration and reinstallation is now essentially complete. Following cooldown and power supply commissioning the first introduction of beam from the Main Injector is expected in mid-April.

\section{ACCELERATOR SCHEDULE}

The current schedule of activities leading up to the initiation of Collider Run II is as follows:

April 1999 Complete Recycler installation (except for stochastic cooling). Initiate beam transfers from Main Injector to Recycler, Tevatron, and Antiproton Source.

May Start Tevatron $800 \mathrm{GeV}$ fixed target run.

November Complete Tevatron $800 \mathrm{GeV}$ fixed target run.

December Complete installation of Debuncher and Recycler stochastic cooling systems.

Start collider changeover.

February 2000 Collider startup.

\section{BEYOND RUN II}

Initial goals for Tevatron Collider Run II include a luminosity of $-1 \times 10^{32} \mathrm{~cm}^{-2} \mathrm{sec}^{-1}$, accompanied by a total delivered integrated luminosity of $2 \mathrm{fb}^{-1}$ by the end of
2002. Improvements in performance beyond these values will require increasing the number of antiprotons in the collider and controlling the beam-beam forces experienced by the antiprotons as the number of proton bunches grows. A number of development projects currently underway at Fermilab are targeting these two areas.

\subsection{Improved Antiproton Availability}

Increasing the number of antiprotons in the collider beyond the quantities given in Table 1 requires the production and storage of more antiprotons. Fortunately the improvements to the Antiproton Source cooling systems described above are sufficient to support a factor of three increase in the antiproton flux into that facility. As a result increasing antiproton availability will depend on: 1)producing more antiprotons; and 2)providing a place to store them.

Increasing the antiproton production rate is anticipated as being achieved through increases in the acceptance of the Antiproton Source and in the proton targeting rate for antiproton production. The former is possible because the apertures of the beam transfer line downstream of the antiproton production target and of the Debuncher ring are limited in a few well-known locations. A program of identifying such locations and opening the aperture could yield a $50 \%$ increase in acceptance. In addition a liquid lithium lens development program now underway at the Budker Institute will, if successful, increase the accumulated yield from the target by up to $30 \%$.

Increasing the number of protons on the antiproton production target will require raising the proton intensity delivered from the Main Injector. One possibility that has been studied is a technique called "slip stacking" [5]. This technique, which was first demonstrated at CERN in the 1970s [6], involves stacking beams in longitudinal phase space. Two subsequent Booster batches are injected into the Main Injector and captured at different energies (different radial positions) by subsets of the $\mathrm{rf}$ cavities running one harmonic number above and below the nominal harmonic number of the accelerator $(h=588)$. The two batches "slip" with respect to each other and are recaptured in the $h=588$ system once they are aligned with each other. A simulation indicates that the beam intensity can be nearly doubled via this process. Of course, the longitudinal beam emittance is also doubled in the process.

The combination of Antiproton Source aperture improvements and slip-stacking should lead to a factor of approximately three increase in the antiproton stacking rate. Unfortunately, the stochastic cooling systems in the Recycler Ring will not be capable of supporting the increased flux coming from the Antiproton Source and so implementation of electron cooling will be required. 


\subsection{Electron Cooling}

An R\&D program aimed at providing electron cooling of $8.9 \mathrm{GeV} / \mathrm{c}$ antiprotons is currently underway at Fermilab [7]. The electron beam requirements for such a system include a $4.4 \mathrm{MeV}$ beam energy and a current of $200 \mathrm{~mA}(\mathrm{dc})$. The electron beam is to be provided by a 5 MV electrostatic generator. Because of the inability of the generator to source more than a few hundred watts, highly efficient beam recovery is a important feature of any such system. The program to date has concentrated on definition of required electron beam properties and the achievement of a sustained high dc current in a $1.5 \mathrm{MV}$ electrostatic generator (known as a "Pelletron") at the National Electrostatics Corporation in Middleton, Wisconsin. The program at NEC is nearly complete, with sustained currents of 200-500 mA demonstrated, and procurement of a $5 \mathrm{MV}$ electrostatic generator is in process. It is anticipated that a demonstration electron beam line could be assembled in 2001, with implementation in the Recycler possible in late 2002.

\subsection{Electron Beam Compensation}

Once the number of proton bunches in the Tevatron rises to the vicinity of 100 , the impact on the antiprotons in the collider, even in the presence of separated orbits, is significant. Each antiproton bunch experiences more than 200 long-range beam-beam encounters with high intensity proton bunches in addition to the two head-on encounters at the interaction points. Because of the lack of uniformity of proton populations in all possible buckets, bunch-tobunch variations in closed orbit, tune, and tune spread can be induced in the antiproton beam. If these variations become too large performance will suffer. Minimization of the bunch-to-bunch variations is currently foreseen as being achieved through control of the proton bunch configuration in the collider.

However, an alternative approach is under study that relies on a low energy electron beam, current modulated at the bunch crossing frequency $(7.5 \mathrm{MHz})$ in order to provide an additional focusing force that cancels the lowest order (tune variation) effect within the antiproton beam [8]. A system consisting of a $6.7 \mathrm{KV}, 3 \mathrm{~A}$ electron beam has been constructed and tests are now underway to demonstrate the ability to program current pulses at 7.5 $\mathrm{MHz}$. If successful implementation could occur in the Tevatron as early as 2001 .

\section{SUMMARY}

After a six year construction period the Main Injector is now complete and the Recycler Ring is nearing completion. Commissioning of the Main Injector has been underway since October 1998 and the accelerator is currently performing at about $60 \%$ of its ultimate design goal. Significant reconfigurations of the Antiproton Source and Tevatron are also nearly complete in anticipation of the reinitiation of collider operations in early 2000 . The Fermilab goal is to operated the collider at an energy of $2000 \mathrm{GeV}$ (center-of-mass) and a luminosity in the range $0.5-1 \times 10^{32} \mathrm{~cm}^{-2} \mathrm{sec}^{-1}$, accumulating a total of $2 \mathrm{fb}^{-1}$ of integrated luminosity by the end of 2002 . Further improvements to collider performance are possible based on increasing the availability of antiprotons and control of the beam-beam interaction. Possible improvements under study include Antiproton Source aperture improvements, a higher gradient lithium lens, slip-stacking in the Main Injector, electron cooling of antiprotons at $8.9 \mathrm{GeV} / \mathrm{c}$, and electron beam compensation of the beam-beam tune shift. Implementation of these new technologies affords the possibility of achieving luminosities in excess of $1 \times 10^{32} \mathrm{~cm}^{-2} \mathrm{sec}^{-1}$ before the LHC becomes operational in the middle of the next decade

\section{REFERENCES}

[1] Stcphen D. Holmes, Status of the Main Injector and Reycler, Proceedings of the 1997 Particle Accelerator Conference, Vancouver.

Information is also available at http://www-fmi.fnal.gov

[2] G. Jackson, editor, The Fermilab Recycler Ring Technical Design Report, Fermilab-TM-1991

[3] S. O'Day, The Fermilab Accumulator Ring Lattice Upgrade, Proceedings of the 1997 Particle Accelerator Conference, Vancouver.

[4] D. McGinnis, The $4-8 \mathrm{GHz}$ Stochastic Cooling Upgrade for the Fermilab Debuncher, Paper submitted to this conference.

[5] S. Shukla et al, Slip Stacking for the Fermilab Luminosity Upgrade, Proceedings of the 1997 Particle Accelerator Conference, Vancouver.

[6] D. Boussard and Y. Mizumachi, Production of Bearns with High Line-Density by Azimuthal Combination of Bunches in a Synchrotron, CERN-SPS/ARF/79-11; 1979 IEEE Particle Accelerator Conference (1979).

[7] S. Nagaitscv, Status of the Fermilab Electron Cooling Project, Paper submitted to this conference.

[8] V. Shiltsev et al, Compensation of Beam-Beam Effects in Tevatron Collider with Use of Electron Beam. Paper submitted to this conference. 